\title{
INSTABILITIES OF MAGNETIC FIELDS IN STARS
}

\author{
R. J. TAYLER \\ Astronomy Centre, University of Sussex, England
}

\begin{abstract}
It has been shown (Markey and Tayler, 1973; Tayler, 1973; Wright, 1973) that a wide range of simple magnetic field configurations in stars are unstable. Although the ultimate effect of the instabilities is unclear, it seems likely that they would lead to enhanced destruction of magnetic flux, so that magnetic field decay would be much more rapid than previously supposed. Instability is almost certain in a nonrotating star containing either a purely toroidal field or a purely poloidal field, which has closed field lines inside the star. In both cases the instability resembles the well known instabilities of cylindrical and toroidal current channels, modified by the constraint that motion must be almost entirely along surfaces of constant gravitational potential.
\end{abstract}

If both toroidal and poloidal fields are present, the problem is more complicated. In a toroidal plasma with a helical field, the worst instabilities are also helical but it is impossible for a helical disturbance to be parallel to a surface of constant gravitational potential everywhere. As a result, the admixture of toroidal and poloidal fields has a stabilizing influence, but it is not at present clear whether the majority of such configurations are completely stable.

The effect of rotation has not yet been studied but it will certainly be important if the rotation period is less than the time taken for an Alfvén wave to cross the region of interest. This is true in most stars unless the internal magnetic field is very much stronger than any observed field.

\section{References}

Markey, P. and Tayler, R. J.: 1973, Monthly Notices Roy. Astron. Soc. 163, 77.

Tayler, R. J.: 1973, Monthly Notices Roy. Astron. Soc. 161, 365.

Wright, G. A. E. : 1973, Monthly Notices Roy. Astron. Soc. 162, 339.

\section{DISCUSSION}

Schatten: I would like to ask either of the last two speakers whether or not they could come up with a complete computer model for their proposed mechanisms for creating fields in the solar core, because one can either neglect magnetic fields and do a complete computer analysis which is incorrect or one can include magnetic fields and must resort to some sort of physical argument.

Tayler: Sorry, I haven't quite understood whether that was a question or a statement.

Schatten: A question. Whether or not you can actually provide calculations which support your views.

Tayler: Oh. The calculations of the existence of the instability are complete. The calculations of the development of the instability are non-existent, but linear calculations of the existence of the instability are complete. 